\title{
Leukemia and Periodontal Health
}

\author{
Bann AlHazmi ${ }^{1}$
}

BDS, MSc

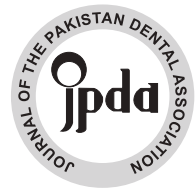

Leukemia is a malignant neoplasm that arises from hematopoietic cells. leukemia has high prevalence among Saudi and Pakistani populations (6.2\% and $4.1 \%$ respectively). Dentists should perceive the serious complications of leukemia and its therapies and to manage leukemic patients in dental practice safely and effectively.

Oral manifestations of hematological malignancies may represent the initial sign of the underlying hematopoietic disease. 65\% of leukemia lesions have some form of oral manifestations.

Accordingly, the purpose of this review is to summaries all the general manifestations of Leukemia as well as the oral manifestations to facilitate early diagnosis and referral.

Leukemia oral manifestations could be pale mucosa or spontaneous bleeding gingiva or bruising and petechiae in the hard and soft palate. Gingival hyperplasia, ulcerations, and opportunistic infections with Candida albicans and Herpes Viruses can occur in oral mucosa.

Dental care for leukemic patients should focus on trauma prevention and meticulous oral hygiene. Antibiotics should be used to prevent and to treat infections (bacterial, virus and fungal infections). Periodontal surgeries like gingivoplasty procedures are considered elective treatments before the diagnosis and or treatment of leukemia and it should not be performed until the patient completes and maintains their antineoplastic treatment. Dentists plays an essential role in the early diagnosis of the leukemia; therefore, they should lead a proper investigation and referral to reach proper diagnosis.

KEYWORDS: Gingival Bleeding, Leukemia, Oral Manifestations, Periodontal Surgeries.

HOW TO CITE: AlHazmi BA. Leukemia and periodontal health. J Pak Dent Assoc 2021;30(1):61-65.

DOI: https://doi.org/10.25301/JPDA.301.61

Received: 12 August 2020, Accepted: 27 November 2020

\section{INTRODUCTION}

$\mathrm{T}$ here are many types of malignant tumors and Leukemia is one of them. Leukemia originates from abnormal hematopoietic cells, with abnormal function, and it has different types. In 2018, the number of Leukemic patients around the world reached 437, 033 cases and it is expected to rise to 23.6 million case per year by 2030. ${ }^{1}$ In Saudi Arabia, leukemia is ranked the 5th among all cancers $\left(7.6 \%\right.$ in males and $4.4 \%$ in females). ${ }^{2}$ The prevalence of children (age less than 14 years) with Leukemia is $38.8 \%$ in 2017. Leukemia has the highest prevalence in Saudi children among other cancers. ${ }^{2}$ Leukemia also ranked the 5th most frequent cancer in Pakistan by new cases and deaths, and it constitutes about $4.1 \%$ of total cancer cases (in both sexes and all ages) in $2018 .{ }^{3}$ The five-year prevalence for leukemic cases in Pakistan is 15980, and the male patients are more prevalent than female $(59.7 \%$ and $40.3 \%$ respectively). ${ }^{3}$

This high leukemic prevalence should draw the attention of health care providers. It is recommended for dentists to perceive the serious complications of leukemia and its therapies and to manage leukemic patients in dental practice

Lecturer, Department of Periodontics and Community Dentistry, College of Dentistry, King Saud University, Riyadh, Saudi Arabia.

Corresponding author: "Dr. Bann A. Al Hazmi” < budont@yahoo.com > safely and effectively. The US National Cancer Institute, necessitates to manage leukemic patients with multidisciplinary approach. The multidisciplinary therapy team should have professionals from oncology, radiation, nutritional, dental, hematology, social and other departments. The dental professionals (generals and specialists) are important to diagnose and treat and prevent oral complications in leukemic patients.

Oral manifestations of hematological malignancies could be the first sign of the underlying hematopoietic abnormalities. Stafford et al. reported that early diagnosis of large numbers of acute non-lymphocytic leukemias cases was a contribution of dentists. ${ }^{5} 65 \%$ of leukemias have some form of oral pathology manifestations. ${ }^{5}$ The common general manifestations clinically are fatigue, weight loss, night sweats, pyrexia, anorexia, pallor, pruritus, splenomegaly, and hepatomegaly. ${ }^{6}$

Accordingly, the purpose of this review is to summaries all the general manifestations of Leukemia as well as the oral manifestations to facilitate early diagnosis and referral.

\section{ORAL MANIFESTATIONS}

Orofacial manifestations, especially in acute leukemia cases, have clinical appearance basically due to leukemic cells infiltration into the oral soft and hard tissues and bone 
marrows. The side effects of leukemia treatments are considered secondary and tertiary effects. For example, recurrent aphthous-like ulcers and periodontal disease are mostly associated with qualitative defects in granulocytes. This defect is one of the chemotherapy side effects.

Oral mucosa: the oral mucosa could look pale or erythematous. The pale mucosa is due to anemia. Bleeding of oral mucosa and blue petechia seen in the palate, tongue or lips are common. The mucosal bleeding is a result of thrombocytopenia. ${ }^{7}$ Ulcerations and infections with Candida albicans and Herpes Viruses can occur in oral mucosa because of immunosuppression condition. ${ }^{8}$

Gingiva: gingival hyperplasia especially in interdental papilla or gingival margins is common in acute cases. The hyperplasia is a result of inflammation or leukemic cell infiltration and it could be localized or generalized. ${ }^{9}$ On the other hand, chronic leukemic cases have lesser infiltration and the gingiva would be paler. ${ }^{9}$ Severe thrombocytopenia (the platelet count is lower than 20,000 cells $/ \mathrm{mm} 3$ ) can cause gingival bleeding. ${ }^{10}$ However, if the thrombocytopenia platelet count is above 20,000 cells $/ \mathrm{mm} 3$, gingiva and palate manifest bruising and petechiae. ${ }^{10}$

Teeth: changes in teeth are not a primary cause changes but a treatment side effects. Inability to maintain good oral hygiene, tendency to eat soft diet and changes in oral microflora habitat are the main reason of cervical teeth caries. Bone leukemic cell infiltration mostly in acute cases, is responsible for teeth displacement and destruction of alveolar bone and periodontal ligaments. ${ }^{5,11}$ Another possible manifestation is tooth pain, and it is due to pulp infiltration.

Bone: bony masses in mandible and maxilla can occur in leukemic patients, however, their incidence is rare. ${ }^{12}$ There are four reported cases with bony masses in oral cavity, three are B-lymphoblastic lymphoma and one T-lymphoblastic leukemia/lymphoma. ${ }^{13}$ These bony masses may look as one hard swelling or multiple lobulated friable mass. On X-rays, these bony masses appear as lytic or sclerotic bone alterations and resemble bone tumors. ${ }^{13}$ Leukemic patients may experience pain or paresthesia in the jaws area. Radiographically, there are loss of lamina dura, changes in periodontal space, resorption of the alveolar bone, and destruction of the bone structure. ${ }^{14}$ All these symptoms are related to leukemic cell infiltrations.

There are secondary and Tertiary complications related to leukemias. The side effects of antineoplastic treatments like radiation therapy or chemotherapy are considered secondary complications. Examples of secondary complications are the complications related to thrombocytopenia, granulocytopenia, and anemia. ${ }^{15}$ The tertiary complications are due to an interaction between therapy itself, the therapy's side effects, and a systemic condition emerges from that therapy. Taste alteration, skin desquamation, candidiasis, xerostomia, dysphasia, opportunistic infections, permanent taste loss, salivary flow reduction, osteoradionecrosis, chondronecrosis and trismus are examples of tertiary complications. ${ }^{15}$

\section{MANAGEMENT OF LEUKEMIC ORAL MANIFESTATIONS}

Treatment of oral manifestations in patients with leukemia is complicated and depends on case stability and prognosis of the disease. Therefore, dental management for leukemic patients should focus on trauma prevention like traumas from ill-fitting dentures or faulty fixed prosthesis, bleeding elimination and prevention and treatment of any oral infection related to leukopenia.

Meticulous oral hygiene practices (teeth brushing, fluoride application, and low-sugar food) should also be stressed before, through and after treatment. ${ }^{16}$

For example, the manifestation of gingival enlargement is managed by meticulous oral hygiene, use of soft toothbrush and antiseptic mouth wash like chlorhexidine $0.12 \%$ twice daily. Oral ulceration can be treated with topical corticosteroid gel (fluocinonide $0.1 \%$, Apply thin layer each day or every 12 hours) and biopsy if necessary. Antibiotic is recommended for ulcers to prevent bacterial infections. Secondary infections are common; therefore, antibiotics should be administered in therapeutic rather than prophylactic doses. ${ }^{16,17}$ Antifibrinolytic mouth rinse is recommended to manage gingival bleeding, as well as good plaque control. ${ }^{18}$ All infections foci must be eradicated completely, by antibiotics or antivirals or antifungals as needed. These medications are to be used as prevention or treatment. ${ }^{18}$

\section{General Considerations Regarding Dental Management in Leukemic patients}

Dental management for leukemic patients is provided through three different stages: 1) pre-antineoplastic treatment assessment and preparation of patients, 2) oral health care during treatment, 3) post-treatment care. ${ }^{9,19}$

\section{Pre-Antineoplastic Treatment Assessment and Patient Preparation:}

Dental care in this stage is directed to treat emergencies and urgent needs only. Elective treatment should be suspended until the case gets stable clinically and laboratory. It is quite important to educate leukemic patients or their relatives the 
practice of meticulous oral hygiene and how it is always essential to reduce oral problems and discomfort. ${ }^{4,18}$ Also, patients must be informed about possible side effects that may arise from antineoplastic therapy in oral cavity such as mucositis and oral pain. Dentists should focus on prevention of oral infections and injuries like sharp denture edges and orthodontics injuries.

Treatment of oral mucosa lesions, carious teeth, endodontic and periodontal diseases, replacing teeth with good fitting dentures and temporomandibular joint dysfunction are examples of treatment that can be done at this stage. ${ }^{4}$

\section{Oral Health Care during Antineoplastic Treatment:}

Patients in this stage are classified as high-risk, because of infections possibility and its serious consequences in patients' immunosuppressed condition. Any oral infection can be fatal or at least worsen the general health status of patients. ${ }^{20}$ The main goals of any dental management at this stage are to keep optimal oral health, to treat the therapy side effects, and to eradicate oral infections. ${ }^{4}$

In this stage there are common chemotherapy side effects. Main oral side effects could be high caries rate, gingival bleeding, gingival abscess, salivary gland dysfunction, herpetic gingivo stomatitis, xerostomia. ${ }^{4}$

There is no restriction to do dental examination, radiographs, prophylaxis and supragingival scaling during antineoplastic treatment phase. ${ }^{17}$

\section{Post-Antineoplastic Treatment Oral Health Care:}

After the completion of leukemia therapy, patients can be treated normally. Patients completed their antineoplastic therapy, and usually there are no oral manifestations neither from illness nor from treatment. However, children who received chemotherapy during tooth formation, could present with enamel demineralization and abnormal dental roots. ${ }^{15}$

Elective dental treatment regimens are allowed. ${ }^{17}$ However, antibiotics prophylaxis is mandatory for at least six months post-treatment. All surgical dental procedures should be covered with antibiotics prophylaxis. ${ }^{21}$ Orthodontic therapy is allowed only after two years of post-therapy and cancer free condition. ${ }^{17}$

The dental treatment must be planned according to the disease and therapy status. Patients in good status with acceptable immune condition can receive more invasive dental procedure. However, the invasive procedures must be done after consultation with hematologist, and platelet count and neutrophil count laboratory tests must be evaluated. ${ }^{9}$

\section{Periodontal Procedures at Different Stages of Leukemic therapy}

The periodontal therapy should aim to treat and avoid any infections, or bleeding. It is of quite importance to consider some hematological indices like neutrophils and platelets counts before any periodontal procedure. Noninvasive procedures do not require special precautions and can be done at any stage of the therapy or disease. For example, clinical examination, radiographic imaging, oral hygiene practices, supragingival scaling and prophylaxis, are noninvasive procedures. ${ }^{17}$

According to Haytac et $\mathrm{al}^{22}$ performing periodontal probing, root planing or extractions requires a neutrophil number of $1,500 / \mathrm{mm} 3$ and platelets number of 40,000 cells/mm3 or above. Periodontal procedures must be performed before the chemotherapy (at least three days); and before the numbers of granulocyte falls below 500 cells $/ \mathrm{mm} 3$. In case of lower hematological indices, dental treatment should be postponed. ${ }^{22}$

Subgingival scaling and root planing and teeth extraction are restricted in pre- and during cancer therapy. Special care must be taken, and antibiotic prophylaxis are needed when subgingival scaling and root planing or extraction are to be performed. Platelets and neutrophils count are important to be assessed with the hematologist. ${ }^{17,21}$

Leukemic patients who received bone marrow transplantation, usually after chemotherapy, need six to twelve months to rehabilitate their immune systems. Therefore, all dental procedures including scaling and root planing should be postponed. ${ }^{4}$

Periodontal surgeries like: gingivoplasty and gingivectomy procedures, flap surgery, teeth extraction, implants placement, and mucogingival surgeries are elective procedures. All elective procedures should not be conducted during the first or second stages (diagnosis and treatment of leukemia). They can be conducted after patients complete the treatment course successfully and restore their health. ${ }^{17,21}$

Koulocheris et $\mathrm{al}^{22}$, state that in oral surgical procedures, the benefit/risk to the patient must be considered, as well as the consequences of chemotherapy cycles; these procedures should therefore be planned and agreed on an interdisciplinary level.

In case of spontaneous oral bleeding, the dentists must control it by using the local measures and improving the oral hygiene. If the bleeding persists, platelet transfusion may be required.

The local measures recommended to stop oral bleeding could be vasoconstrictor agents or clots or tissue guards. Epinephrine or collagen hemostatic agents can be applied to reduce the blood flow from bleeding vessels. Topical thrombin is effective to organize and stabilize blood clots. To protect the organized blood clots, the use of mucosa adhesive products, such as those based on cyanoacrylate, is effective. ${ }^{17}$ 
There was a cross-sectional study done to evaluate the relation between the periodontal status of patients with leukemia and their hematological parameters like platelets and leukocytes count. Periodontal indices for patients were assessed. The indices are plaque index (PII), gingival index (GI), probing depth (PD), bleeding on probing (BOP) and clinical attachment loss (CAL). There was no significant correlation between periodontal and hematological parameters. It was concluded that periodontal status was related to plaque level and it did not correlate with hematological parameters regardless of the leukemia type. ${ }^{23}$

Oral hygiene must be maintained for leukemic patients using plaque control measures like teeth brushing, flossing and mild antimicrobial mouthwashes. The antiseptic mouthwashes stimulate ulcer healing and minimize the incidence of infections. However, if there is evidence of oral infection, high-risk patients should be given broadspectrum antibiotics intravenously. ${ }^{9}$

\section{CONCLUSIONS}

Dental professionals should be qualified to notice and recognize oral signs of underlying systemic conditions. This would help for early diagnosis and better prognosis of the condition. Good dental maintenance for leukemic patients is essential part in their general treatment. Part of dental maintenance is oral hygiene practice and patient education of its importance. Keeping good oral hygiene improves patient comfort and prevent many complications during antineoplastic treatment coarse. Dental management for active leukemic patients is restricted for urgent noninvasive procedures, elective periodontal treatment is delayed until patient condition cured and stable. Consultation with oncologist and laboratory investigation like platelet count, neutrophil count, and INR before any dental treatment performed to leukemic patients are proper dental management.

\section{ACKNOWLEDGMENT}

None.

\section{CONFLICTS OF INTEREST}

There are no conflicts of interest.

\section{REFERENCES}

1. Global Cancer observatory. International Agency for Research on Cancer. Switzerland: WHO; 2018 [cited 2019 Oct 16]. Available from: https://gco.iarc.fr/.
2. SCR. Cancer Incidence Report. Saudi Arabia: Saudi Cancer Registry/ Ministry of Health; 2018, [cited 2019 Oct 15]. Available from: https://www.shc.gov.sa/ar/NCC/Activities/.

3. Global Cancer observatory. International Agency for Research on Cancer. Switzerland: WHO; 2018 [cited 2020 Sep 13]. Available from: https://gco.iarc.fr/today/data/factsheets/populations/586-pakistanfact-sheets.pdf

4. US National Cancer Institute. Oral Complications of Chemotherapy and Head/Neck Radiation. Maryland: The National Institutes of Health; 2011 [cited 2019 Oct 17]. Available from:

https://www.cancer.gov/types/leukemia .

5. Adeyemo TA, Adeyemo WL, Adeniran A, Akinbami AJ, Akanmu AS. Orofacial manifestation of hematological disorders: Hematooncologic and immuno-deficiency disorders. Ind J Dent Res, 2011; 22:688-97

https://doi.org/10.4103/0970-9290.93458

6. Prajapati Z, Kokani MJ, Gonsai RN. Clinicoepidemiological profile of hematological malignancies in pediatric age group in Ahmedabad. Asian J Oncolog. 2017;3:54-8.

https://doi.org/10.4103/2454-6798.209330

7. Lim HC, Kim CS. Oral signs of acute leukemia for early detection. J Periodontal Implant Sci, 2014; 44:293-99.

https://doi.org/10.5051/jpis.2014.44.6.293

8. Neville B, Damm D, Allen C, Bouquot J. Hematologic disorders. In: Oral and Maxillofacial Pathology. St. Louis, Missouri: Saunders/Elsevier, 3rd edition, 2009: 573-613.

9. Little JW, Falace DA, Miller CS, Rhodus NL. Disorders of white blood cells. In: Dental Management of the Medically Compromised Patient. St. Louis, Missouri: Elsevier, Mosby, 1997:373-95.

10. Epstein JB, Vickars L, Spinelli J, Reece D. Efficacy of chlorhexidine and nystatin rinses in prevention of oral complications in leukemia and bone marrow transplantation. Oral Surg Oral Medic and Oral Path, 1992;73:682-89.

https://doi.org/10.1016/0030-4220(92)90009-F

11. Nasim YS, Shetty YR, Hegde AM. Dental health status in children with acute lymphoblastic leukemia. J Clin Pediatr Dent, 2007; 31:21013.

https://doi.org/10.17796/jcpd.31.3.73mu542187l75700

12. Epstein JB, Priddy RW, Sparling T, Wadsworth L. Oral manifestations in myelodysplastic syndrome. Review of the literature and report of a case. Oral Surg Oral Med Oral Pathol, 1986; 61:46670.

https://doi.org/10.1016/0030-4220(86)90389-0

13. Talreja KL, Barpande SR, Bhavthankar JD, Mandale MS. Precursor B-cell lymphoblastic lymphoma of oral cavity: A case report with its diagnostic workup. J Oral Maxillofac Pathol, 2016; 20:133-136. https://doi.org/10.4103/0973-029X.180973

14. Brazelton J, Louis P, Sullivan J, Peker D. Temporomandibular 
joint arthritis as an initial presentation of acute myeloid leukemia with myelodysplasia-related changes: a report of an unusual case. J Maxillofac Surg. 2014; 72:1677-83. https://doi.org/10.1016/j.joms.2014.02.007

15. Girish Babu KL, Mathew J, Doddamani GM, Narasimhaiah JK, Naik LR. Oral health of children with acute lymphoblastic leukemia: A review. J Orofac Sci. 2016; 8:3-11.

https://doi.org/10.4103/0975-8844.181915

16. Stafford R, Sonis S, Lockhart P, Sonis A. Oral pathoses as diagnostic indicators in leukemia. Oral Surgery Oral Med Oral Pathol. 1980; 50: 134-39.

https://doi.org/10.1016/0030-4220(80)90200-5

17. Zimmermann C, Meurer MI, Grando LJ, Gonzaga Del Moral JÂ, da Silva Rath IB, Schaefer Tavares S. Dental treatment in patients with leukemia. J Oncol. 2015; 2015:571739.

https://doi.org/10.1155/2015/571739

18. Francisconi CF, Caldas RJ, Oliveira Martins LJ, Fischer Rubira CM, da Silva Santos PS. Asian Pac J Cancer Prev, 2016;17:911-15. https://doi.org/10.7314/APJCP.2016.17.3.911

19. Elad S, Raber-Durlacher JE, Brennan MT, Saunders DP, Mank AP, Zadik Y, et al. Basic oral care for hematology-oncology patients and hematopoietic stem cell transplantation recipients: a position paper from the joint task force of the Multinational Association of Supportive Care in Cancer/International Society of Oral Oncology (MASCC/ISOO) and the European Society for Blood and Marrow Transplantation (EBMT). Support Care Cancer, 2015;23:223-36.

https://doi.org/10.1007/s00520-014-2378-x

20. Tong DC, Rothwell BR. Antibiotic prophylaxis in dentistry: a review and practice recommendations. J Am Dent Assoc, 2000;131:36674.

https://doi.org/10.14219/jada.archive.2000.0181

21. Koulocheris P, Metzger MC, Kesting MR, Hohlweg-Majert B. Life-threatening complications associated with acute monocytic leukaemia after dental treatment. Aust Dent J, 2009;54:45-8. https://doi.org/10.1111/j.1834-7819.2008.01087.x

22. Haytac MC, Dogan MC, Antmen B. The results of a preventive dental program for pediatric patients with hematologic malignancies. Oral Health Prev Dent, 2004;2:59-65.

23. Angst PD, Dutra DA, Moreira CH, Kantorski KZ. Periodontal status and its correlation with haematological parameters in patients with leukaemia. J Clin Periodontol, 2012;39:1003-010. https://doi.org/10.1111/j.1600-051X.2012.01936.x 